\title{
Cambios observados y proyectados en los tipos de regímenes bioclimáticos de Argentina
}

\section{Observed and projected changes in the types of bioclimatic regimes in Argentina}

\author{
Valeria Soledad Duval \\ valeria.duval@uns.edu.ar @ 0000-0001-9048-3058 \\ Universidad Nacional del SUr-CONICET \\ 12 de Octubre.1098. CP 8000 Bahía Blanca, Argentina \\ Rafael Cámara-Artigas \\ rcamara@us.es @ 0000-0003-1046-3749
}

Departamento de Geografía Física y Análisis Geográfico Regional, Facultad de Geografía e Historia, Universidad de Sevilla. C/ Doña María de Padilla s/n. 41004 Sevilla, España

\section{INFO ARTÍCULO}

Recibido: 23/10/2021

Revisado: 03/12/2021

Aceptado: 12/01/2022

\section{PALABRAS CLAVE}

Cartografía bioclimática

Cambio climático

Ecorregiones

Geografía física

\section{KEYWORDS}

Bioclimatic mapping

Climate change

Ecoregions

Physical geography

\begin{abstract}
RESUMEN
El estudio de los límites bioclimáticos es esencial en cada región para conocer la distribución de las formaciones vegetales. El objetivo del trabajo fue elaborar mapas bioclimáticos de Argentina en tres períodos de tiempo (Ultimo Máximo Glaciar, actualidad y escenario de cambio climático RCP8.5 del 2070) y analizar sus cambios. Para ello, se aplicó la metodología de los tipos de regímenes bioclimáticos a partir de bases de datos continuas climáticas de WordClim. Como resultado se observó una reducción en la superficie de los tipos térmicos mesocriófilo, hipercriófilo y criófilo, entre el Último Máximo Glaciar y la actualidad y aparece el termófilo en el escenario futuro. Esta investigación es una aportación para la comprensión de la distribución de la vegetación a escala nacional y su dinámica.
\end{abstract}




\section{INTRODUCCIÓN}

El clima es el principal elemento que determina la distribución de la vegetación en el mundo (Cain, 1944; Tuhkanen, 1980). El bioclima, por su parte, tiene en consideración las necesidades térmicas e hídricas de las plantas integrantes en una formación vegetal. La limitación en algunos de estos aspectos es clave para explicar la distribución de dichas formaciones. Es por ello que es necesaria la caracterización y la cartografía de las condiciones bioclimáticas para comprender la distribución actual de las formaciones vegetales del planeta. Dicha distribución se verá matizada localmente por las condiciones geobotánicas que rijan: así sobre el karst o sobre formaciones de costras calcáreas o férricas, o los procesos de saturación de agua por inundación, marcarán cambios por defecto o exceso de las condiciones hídricas, dando lugar a formaciones intrazonales, como las marismas, los manglares, sabanas inundables, los esteros por exceso de agua, o las sabanas o estepas por la presencia de formaciones superficiales específicas.

El conocimiento preciso y detallado de la composición y distribución vegetal en conjunto con la disponibilidad de datos climáticos y de Sistemas de Información Geográfica, para su análisis, contribuyen a delimitar las fronteras/ecotonos bioclimáticos y a elaborar modelos que permitan definir programas para la conservación de las especies y de sus hábitats. Además, posibilita la determinación de escenarios futuros de cambio climático y por ende de los límites fitogeográficos de una región (Fernández, 2004).

Un importante avance en la bioclimatología se generó a partir de la creación de índices que contribuyeron a definir las clasificaciones bioclimáticas, entre ellas la propuesta por Holdridge (1947) sobre la base de las zonas de vida, la de Walter (1977) en los diagramas ombrotérmicos del atlas climatológico y el más actual de Bailey (2009), que considera las ecorregiones con un propósito de conservación estableciendo distintas escalas de análisis. Por otra parte, en Argentina, Bruniard (2000) también realizó una aproximación al modelo fitoclimático mundial. Este se basó en el establecimiento de valores-límite críticos para el desarrollo de las diversas comunidades vegetales sobre la base de los regímenes térmicos e hídricos. El autor definió 7 valores críticos de temperatura que le permitieron determinar 9 zonas caracterizadas por su régimen térmico anual y 2 índices de humedad (anual y estival). De la combinación de ambos, se identificaron 20 tipos de vegetación de acuerdo a su fisonomía y ritmo estacional. Los resultados se mostraron en mapas a escala continental, aunque dicho modelo se ajusta más precisamente en el continente americano y no así a los restantes (Fidalgo \& Muñoz, 2003).

En Argentina, no existe una profundización y/o avances actuales sobre una clasificación bioclimática del territorio. Tampoco hay estudios realizados sobre la temática a escala nacional, en el pasado y en un futuro. Si existe, sin embargo, un trabajo detallado de las ecorregiones y complejos ecosistémicos del país, realizado por Morello et al. (2012) en el cual se trata cada ecorregión a escalas entre 1:1.000.000 y 1:300.000 (incluso más pequeñas) desde esta categoría desciende a la subregión, complejo, sistemas ecológicos, tipo de tierra (formaciones vegetales) y fases (vegetación y suelos). En cada ecorregión se trata la geomorfología, clima, ambiente natural y ambiente humano, descendiendo luego a las subregiones, complejos, etc., desglosando apartados en cada uno de ellos. En este trabajo se utiliza el nivel de ecorregión para relacionarla con los tipos de bioclimas, contando con la base cartográfica vectorial del Instituto Geográfico Nacional de Argentina (https://www.ign.gob.ar/NuestrasActividades/InformacionGeoespacial/CapasSIG).

El objetivo del presente trabajo es elaborar una cartografía bioclimática de Argentina en tres períodos de tiempo (Ultimo Máximo Glaciar-20.000 B.P.-, actualidad y escenario de cambio climático RCP-Trayectorias de concentración representativas- 8.5 para el año 2070). Se han elegido estos tres momentos por, en el caso del último Máximo Glaciar, disponer de base de datos de este momento y ser el pulso más frío antes del Holoceno (http://www.worldclim.com/version1). En el escenario del cambio climático se ha optado por una situación extrema (RCP 8.5) en un periodo de tiempo de medio plazo (entre los años 1970-2000). Se seleccionó el modelo CCSM4 (Sistema Climático Comunitario) patrocinado por la National Science Foundation (NSF) y el Departamento de Energía de los Estados Unidos (DOE) porque es un modelo de clima para simular el sistema climático de la Tierra, compuesto por cinco modelos separados de la atmósfera, el océano, la tierra, el hielo terrestre y el hielo marino de la Tierra. Se suma un componente acoplador central que coordina los modelos y pasa la información entre ellos y permite realizar investigaciones fundamentales sobre los esta- 
dos climáticos pasados, presentes y futuros de la Tierra. Se ha utilizado tanto para el Ultimo Máximo Glaciar como para el escenario de cambio climático futuro, y así de esta forma, la comparación de resultados es coherente respecto al modelo. La implementación estándar para las cuadrículas en CCSM ha sido que los modelos de la atmósfera y la tierra se ejecutan en cuadrículas idénticas y el del océano y el hielo marino se ejecutan a su vez también en cuadrículas idénticas.

Esta elección de un escenario extremo de RCP está justificada por lo que se expone a continuación. Hausfather \& Peters (2020) indicaron que el escenario de cambio climático con RCP 8.5 se trata de un futuro improbable ya que se basó en la proyección de una sobreestimación de la producción de carbón. Consideran que para 2070 este tipo de recursos estará en vías de agotamiento y no podrá producirse más emisión de gases invernadero, porque se habrá sustituido por energías limpias alternativas como la solar o la eólica. Según estos autores, esto hacía que el escenario RCP 8.5 sea cada vez más inverosímil con cada año que pasa. Desde el Quinto Reporte del IPCC -Grupo Intergubernamental de Expertos sobre el Cambio Climáticose ha pensado que esta situación es muy poco probable, pero aún posible, ya que las retroalimentaciones no se comprenden bien (Ward et al., 2012). Pero la actual crisis energética ha hecho que muchos países desarrollados vuelvan a explotar sus recursos de minas de carbón, para sustituir al gas y el petróleo como fuentes de energía. Incluso China que como gran potencial industrial ha agotado sus recursos de carbón desde principios del siglo XXI (Zhang et al., 2016) y está demandando carbón actualmente a otros países.

Tal como se observa en la tabla 1, la proyección para 2070 de RCP 8.5 es de $2{ }^{\circ} \mathrm{C}$ de media. El objetivo central del Acuerdo de París fue mantener el aumento de la temperatura mundial en este siglo por debajo de los $2^{\circ} \mathrm{C}$ por encima de los niveles antes de la Revolución Industrial (IPCC, 2013).

Tabla 1. Proyecciones de aumento del calentamiento global $\left({ }^{\circ} \mathrm{C}\right)$.

\begin{tabular}{|c|c|c|}
\hline & $2046-2065$ & $2081-2100$ \\
\hline Escenario & Media y rango probable & Media y rango probable \\
\hline RCP 2.6 & $1.0(0.4$ a 1.6$)$ & $1.0(0.3$ a 1.7$)$ \\
\hline RCP 4.5 & $1.4(0.9$ a 2.0$)$ & 1.8 (1.1 a 2.6$)$ \\
\hline RCP 6 & $1.3(0.8$ a 1.8$)$ & $2.2(1.4$ a 3.1$)$ \\
\hline RCP 8.5 & $2.0(1.4$ a 2.6$)$ & 3.7 (2.6 a 4.8$)$ \\
\hline
\end{tabular}

Fuente: IPCC, 2013.

El trabajo de Steffena et al. (2018) detecta, sin embargo, un umbral en el cual las temperaturas pueden aumentar entre 4 y $5^{\circ} \mathrm{C}$ comparadas con niveles pre-industriales, tomando en cuenta los mecanismos de retroalimentación del sistema climático. Esto avalaría la situación de $2{ }^{\circ} \mathrm{C}$ para un RCP 8.5 en 2070, a la que acompañaría un incremento medio del nivel del mar de 0,30 m para 2070 y de 0,63 m para 2100 (Cámara et al., 2022).

Este trabajo es un aporte esencial debido a la relación existente entre los regímenes bioclimáticos y la distribución de las formaciones vegetales. De esta manera se puede conocer la correlación entre ambas variables. Por otra parte, el análisis multitemporal permite realizar proyecciones sobre el impacto que el incremento de los gases del efecto invernadero (GEI) tiene sobre la distribución de los regímenes bioclimáticos. La base cartográfica contribuye a visualizar los cambios espaciales de estos regímenes en los distintos escenarios temporales.

\section{ANTECEDENTES TEÓRICOS}

Recientemente, Cámara et al. (2020) propusieron una cartografía sobre la base a los tipos de regímenes bioclimático (TRB) representados en tres escalas: mundial, regional y local. Se identificaron en la escala 
local 162 subtipos de regímenes (TRBs) que surgen de la combinación de los 27 tipos de regímenes bioclimáticos, según las limitaciones térmicas e hídricas de la vegetación en cada espacio, con los 9 tipos ombrotérmicos de Thornthwaite. También realizaron cartografías considerando la variable temporal, y es por ello que también es una metodología multitemporal, ya que ha sido aplicada para el período del último Máximo Glaciar y para escenarios futuros de cambio climático. A escala nacional, hay ejemplos de la aplicación de esta metodología en España, tanto para el escenario actual como para el del último Máximo Glaciar. También se han utilizado para estudios de investigación o asesorías internacionales en Mozambique, Guinea Ecuatorial, Brasil, Chile y Estados Unidos (Cámara et al., 2020). A escala regional se empleó en el caso de Paraiba, Brasil (Cámara et al., 2022).

En América Latina, existen algunos países que cuentan con el mapa bioclimático nacional. Entre ellos se destacan México, Chile y Ecuador. En el primer caso, Hernández Cerda et al. (2018) identificaron 7 bioclimas en México comparando dos sistemas de clasificación de distinta escala de aplicación: la climática de Köppen modificado por García (1964) y el Worldwide Bioclimatic Classification System de Rivas Martínez et al. (2011). En Chile, Uribe et al. (2012) realizaron un atlas bioclimático en el cual integraron variables topoclimáticas, de vegetación y de topografía local enfocado al uso agrícola. El Ministerio de Ambiente del Ecuador (2013) actualizó el mapa bioclimático continental del país donde se reconocen 4 grandes bioclimas tropicales, con limitaciones del modelo vinculadas a las zonas de "cordillera costanera". En Perú, Ninahuaman (2016) estableció 12 regiones bioclimáticas para el país sobre la base de la clasificación de Bagnouls \& Gaussen (1963), aporte que se convirtió en un antecedente fundamental a escala nacional. En la mayoría de los casos antes mencionados, existe una base cartográfica de las clasificaciones de los regímenes bioclimáticos.

En Argentina, hay una clasificación bioclimática de todo el territorio publicada por Czajkowski et al. (2009), quienes lo aplicaron en el campo de la conservación energética. Utilizaron como base, los datos de 154 estaciones meteorológicas otorgados por el Servicio Meteorológico Nacional (SMN) del país. De la aplicación y combinación de diferentes variables se obtuvo el mapa final con 5 categorías, las cuales no se nombran y analizan en profundidad. También hay publicaciones con mapas de algunas regiones como el noroeste argentino (Peroné \& Cannelli, 1987) o de algunas provincias como Buenos Aires (Czajkowski \& Rosenfeld, 1992), Mendoza (González et al., 2009) y Jujuy (Entrocassi et al., 2014).

Por otra parte, las grandes transformaciones que se están generando como producto del cambio climático hacen necesario que se elaboren modelos de regímenes bioclimáticos que contemplen escenarios futuros con niveles de concentración de GEI. De esta forma, se puede analizar las tendencias y los cambios que se sucederán en estos regímenes en respuesta a la concentración de estos gases en la atmósfera. En los países latinoamericanos no se han realizado estudios de este tipo a escala nacional. Sin embargo, se registra, a nivel regional, la aplicación en Paraiba (Brasil) donde se elaboró un mapa de los regímenes bioclimáticos para el escenario de cambio climático de 2070, con RCP 8.5 (Cámara Artigas et al., 2022). En Argentina no se ha realizado ningún mapa que muestre ese cambio a futuro, aunque si se registra la aplicación de gráficos y modelados de temperatura y precipitaciones para esos escenarios (Zazulie et al., 2017; Rolla et al., 2018; Doyle, 2019).

\section{METODOLOGÍA}

Para lograr la representación de los TRB se aplicó la metodología de Cámara Artigas et al. (2020), que es un método analítico y cartográfico, que tiene un enfoque multiescalar y multitemporal sobre las formaciones vegetales a partir de los regímenes bioclimáticos. La base de datos climática continua a escala mundial utilizada fue Worldclim (https://www.worldclim.org/data/index.html) para el período glaciar, el escenario actual y el futuro de cambio climático. Los datos son producto de la interpolación de 9.000 a 60.000 estaciones meteorológicas y su resolución espacial es de 1 km² (Hijmans et al., 2005). Los valores mensuales de temperatura y de precipitación fueron descargados seleccionando el modelo climático CCSM 4.0 para el área de análisis. A esta capa de escala planetaria se le realizó un recorte o máscara con los límites del territorio 
argentino en el software ArcGis $10.4{ }^{\circledR}$. Este procedimiento se realizó para las condiciones históricas, las actuales y las futuras, con proyecciones de cambio climático RCP 8.5 para el escenario 2070.

Partiendo de la metodología de balances bioclimáticos de Montero de Burgos \& González (1974) se opera con los rasters de temperatura y precipitación mensual, para obtener las variables necesarias para la identificación de los regímenes bioclimáticos. A partir de ellos, se obtuvieron los rasters mensuales y anuales de paralización vegetativa hídrica y térmica. La temperatura es un factor fundamental en el desarrollo de las plantas, siendo limitante o potenciador de su distribución considerando los límites óptimos de cada especie. Sobre la base de ello, la clasificación utilizada en este trabajo considera la cantidad de meses en el año con presencia o no de paralización vegetativa térmica (PVT). En la tabla 2 se muestra cada categoría (termofilia, euritermofilia, criofilia, mesocriofila e hipercriofilia). De la misma forma, existe una paralización vegetativa hídrica (PVH) que se basa en la presencia y/o ausencia de agua. La PVH se clasifica en ombrofilia, mesofilia, tropofilia, xerofilia e hiperxerofilia, según la cantidad de meses en la cual la planta presenta déficit de agua (tabla 2).

Tabla 2. Clasificación de los tipos de regímenes bioclimáticos (TRB) en función de los meses de la paralización vegetativa térmica (PVT) e hídrica (PVH).

\begin{tabular}{|c|c|c|c|c|}
\hline Meses de PVT & $\mathbf{0}$ & $\mathbf{1}$ a $\mathbf{5}$ meses & $\mathbf{6}$ a $\mathbf{1 0}$ meses & $\mathbf{1 1}$ a $\mathbf{1 2}$ meses \\
\hline TRB & $\begin{array}{c}\text { Termófilia } \\
\text { Euritermofilia }\end{array}$ & Criofilia & Mesocriófilia & Hipercriófilia \\
\hline Meses de PVH & $\mathbf{0}$ & $\mathbf{1}$ a $\mathbf{4}$ meses & $\mathbf{5}$ a $\mathbf{8}$ meses & $\mathbf{9}$ a 12 meses \\
\hline TRB & $\begin{array}{c}\text { Ombrofilia } \\
\text { Mesofilia }\end{array}$ & Tropofilia & Xerofilia & Hiperxerofilia \\
\hline
\end{tabular}

Fuente: Elaboración propia.

Según lo expuesto, los regímenes bioclimáticos tropicales son aquellos cuya media mensual es superior a los $18{ }^{\circ} \mathrm{C}$, mientras que los hipercriófilos son los que poseen al menos 10 meses con temperaturas medias mensuales por debajo de $7,5^{\circ} \mathrm{C}$. En el caso de la humedad, los ombrófilos son los más húmedos y los hiperxerófilos, los de mayor aridez.

Luego de obtener los rasters del Índice Ombrotérmico de Thornthwaite y Mather (1955) y los de PVT y $\mathrm{PVH}$, se generaron los rasters condicionales de tropicalidad, subtropicalidad y zonas templadas y frías de Argentina. La explicación y desarrollo de las fórmulas usando el software ArcGis ${ }^{\circledR}$ se encuentran desarrollados en el anexo de Cámara Artigas et al. (2020). Como resultado se obtuvieron los rasters de los subtipos de regímenes bioclimáticos, donde su nomenclatura se conforma con la denominación térmica seguida de la denominación hídrica y su tipo ombrotérmico (por ejemplo: euritermo mesófilo húmedo medio). En la elaboración de cada mapa se desestimaron aquellos subtipos de regímenes bioclimáticos cuya cantidad de píxeles fuera inferior a 500 (equivalente a $350 \mathrm{Km}^{2}$ o un área equivalente de $18 \times 18 \mathrm{Km}$ ) por ser poco representativo de la superficie del territorio argentino a esta escala. Se les aplicó los colores definidos para cada subtipo (https://www.geografiafisica.org/wp-content/uploads/2020/11/Figura5.jpg), según lo establecido por Cámara et al. (2020) y posteriormente se calculó la superficie (en $\mathrm{km}^{2}$ ) de cada uno de ellos para realizar una comparación más ajustada entre los mapas de cada período (pasado, escenario actual y futuro).

\section{RESULTADOS}

Se realizaron tres mapas de regímenes bioclimáticos a escala nacional para cada periodo seleccionado (Último Máximo Glaciar, escenario actual y futuro con RCP 8.5 para el año 2070), usando la metodología mencionada anteriormente. A continuación, se desarrolla la explicación de la distribución de los regímenes bioclimáticos en escenario temporal y se visualiza su cartografía. 


\subsection{Los Tipos de Regímenes Bioclimáticos durante el Último Máximo Glaciar}

El Último Máximo Glaciar fue la época de máxima extensión de la superficie de hielo durante la Última Glaciación Pleistocena, que tuvo una duración de alrededor de 100.000 años y finalizó hace 11.000 B.P. El nivel de los océanos descendió hasta 100 m en relación con la actualidad y el clima frío de este período dio lugar a la existencia del permafrost en las latitudes medias de casi toda la superficie terrestre. En el actual territorio argentino, el Último Máximo Glaciar tuvo su mayor desarrollo entre los 24.000 y 22.000 B.P., donde el volumen de hielo acumulado fue superior al resto del período, según Ponce \& Rabassa (2012). Durante este período, se incrementó la superficie de la Patagonia continental como resultado de la disminución del nivel del mar, que se encontraba a los $140 \mathrm{~m}$ por debajo del actual, y la exposición de la plataforma. Esto contribuyó a que el efecto moderador marino sea menor dando lugar al aumento de las temperaturas extremas y a la disminución de las precipitaciones (Ponce \& Rabassa, 2012). Se incrementó el volumen de hielo acumulado y se intensificaron los vientos provenientes del oeste, siendo más fríos y secos (Compagnucci, 2011).

La finalización de este período se marcó por el rápido decrecimiento en el volumen del hielo. Su causa se debe a cambios en la radiación solar recibida en el hemisferio Norte hace 22.000 B.P., asociado a los ciclos de Milankovich. Como resultado se desencadenaron transformaciones en el clima planetario, las cuales incidieron directamente en la deglaciación y en el incremento del nivel del mar. De acuerdo a la descripción antes realizada, existía una variedad de paleoambientes. En el mapa de los TRB del Último Máximo Glaciar (mapa 1), se observa una superficie mayor que la actual ya que la plataforma continental, tal como se explicó, fue sobre expuesta en este período, debido al descenso del nivel del mar. Se reconocieron una totalidad de 16 TRB y 64 subtipos.

Durante este período se puede observar que el tipo térmico con mayor superficie era el criófilo, seguido del mesocriófilo y del hipercriófilo. El euritermófilo se desarrollaba en el sector norte, principalmente centro y Este y era el de menor extensión. No hay registro del termófilo para ese período. El TRB crío mesófilo fue el de mayor extensión, el cual se caracteriza por presentar paralización térmica de 1 a 5 meses, aunque no hídrica. Se distribuía principalmente en el centro del actual país y en parte del Mar Argentino. El subtipo que tenía una superficie mayor era el crío mesófilo seco-subhúmedo, también en parte de la actual región noroeste donde se encentraban los regímenes más secos. El crío xerófilo es otro régimen bioclimático de gran expansión al Sur del continente durante este período, principalmente del subtipo crío xerófilo árido. A diferencia del anterior, hay una paralización hídrica de 5 a 8 meses y térmica de 1 a 5 meses.

Otro subtipo con mayor superficie fue el mesocrío xerófilo árido que ocupaba el centro-sur de la Patagonia y noroeste del país, vinculado con las áreas de mayor altura. En relación con los TRB asociados a las zonas polares, el hipercrío mesófilo era el de mayor área y se desarrollaba en el sector oeste, vinculado a la Cordillera de los Andes y en la zona sur, en la actual Tierra del Fuego e Islas Malvinas. El subtipo más representativo fue el hipercrío mesófilo seco-subhúmedo, que se caracteriza por una paralización térmica anual mayor de 9 meses. Además, en este período existía una mayor superficie de regímenes bioclimáticos vinculados con los de tipo mesófilos y xerófilos, en cuestión de humedad. En relación con la temperatura, predominaban los crio y mesocrío.

\subsection{Tipos de Regímenes Bioclimáticos en la actualidad}

En el actual mapa de TRB (mapa 2) se observan cambios en relación con el mapa del Último Máximo Glaciar (mapa 1), tanto en la superficie terrestre como en los regímenes bioclimáticos, como producto del período postglacial. El ascenso del mar, luego de la última glaciación generó que la superficie terrestre se viera reducida. Como primer análisis se observa la existencia de 17 TRB y 75 subtipos. La comparación con el mapa de TRB del Último Máximo Glaciar muestra que algunos de esos TRBs tienen una mayor extensión y, por el contrario, otros se ven reducidos en su área.

En la actualidad, son los eurotermófilos (bioclimas subtropicales) aquellos que ocupan más superficie del país. En el Último Máximo Glaciar, este estaba reducido a la zona centro-Este y actualmente dicho límite 
se extendió hacia el Sur. Se reconoce el euritermo mesófilo como aquel TRB que predomina en centro Este del país. Los subtipos que dominan son el euritermo mesófilo subhúmedo-húmedo y el euritermo mesófilo seco-subhúmedo. Por otra parte, dentro de los criófilos (bioclimas templados-fríos), el crio tropófilo es el que adquiere mayor relevancia siendo el crio tropófilo árido, dentro de los subtipos, el más representativo.

En comparación con el mapa anterior, los TRB críofilos se desarrollan principalmente en la Patagonia. En los mesocriofilos e hipercriofilos (bioclimas subpolares y polares) se observa una reducción de su superficie quedando relegados al Sur del país en la zona de Tierra del Fuego e Islas Malvinas y en la Cordillera de los Andes. Entonces, en síntesis, predominan los euritermofilos y criofilos, en relación con los tipos termófilos (tropicales) que están ausentes, y en cuanto a la clasificación según la paralización hídrica, son más representativos los mesófilos (sin PVH) y tropófilos (con PVH de 1 a 4 meses).

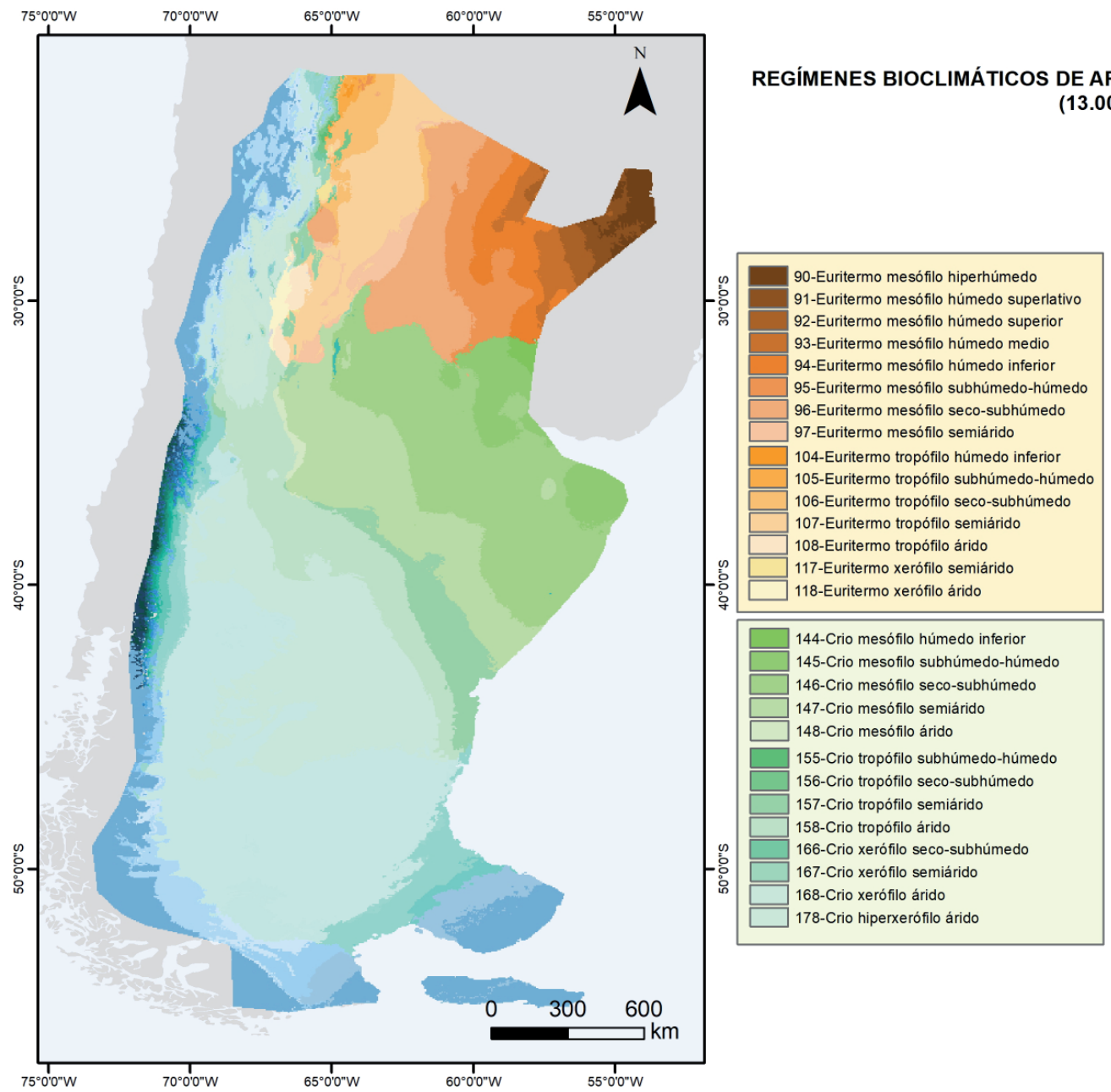

(13.000 BP)

Mapa 1. Tipos de Regímenes Bioclimáticos de Argentina durante el Último Máximo Glaciar. Fuente: Elaboración propia.

En base a esta relación bioclimática, se ha realizado una combinación de las ecorregiones de Argentina. Se utilizó 16 de las 18 ecorregiones identificadas en la obra de Morello et al. (2012) (se ha desestimado el mar Argentino y la Antártida). Hay ecorregiones que responden a situaciones intrazonales como áreas inundadas, como es el caso del Chaco Húmedo, Deltas e Islas y Esteros del Iberá u otras que hacen referencia a situaciones con relieve como Altos Andes, Puna, Yungas, Monte de llanura y mesetas y Monte de sierras y Bolsones. 


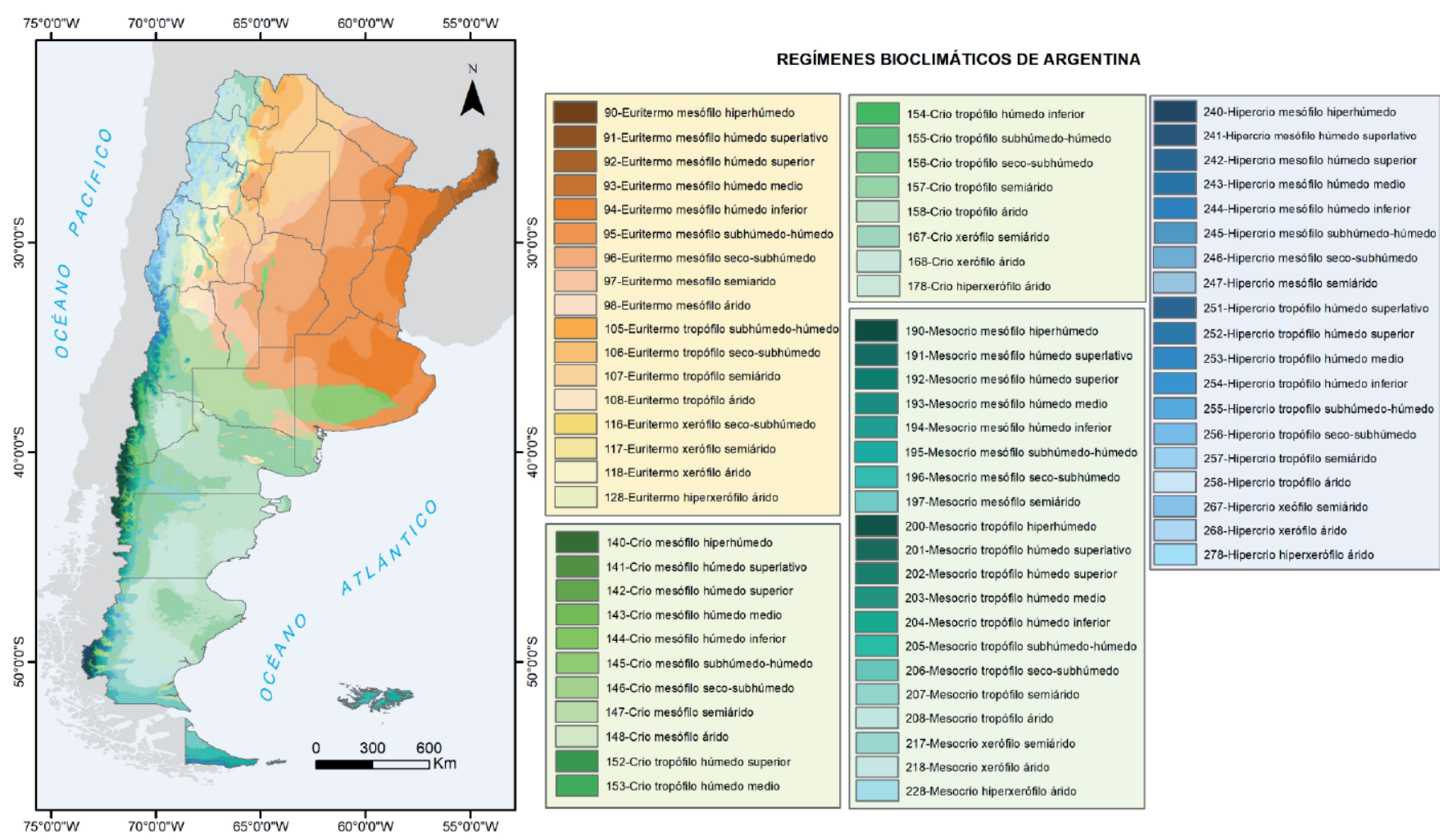

Mapa 2. Tipos de Regímenes Bioclimáticos de Argentina en la actualidad. Fuente: Elaboración propia.

En el dominio subtropical, el TRB que más veces está presente en las ecorregiones es el euritermo mesófilo en sus diferentes situaciones de ombroclimas:

- superlativo a medio en la Selva Paraenense,

- húmedo medio en Campos y Malezales,

- húmedo inferior a húmedo-subhúmedo en los Esteros de Iberá, al norte de La Pampa y el Espinal,

- húmedo inferior a subhúmedo seco en la Pampa,

- húmedo-subhúmedo a subhúmedo-seco en el Chaco húmedo,

- subhúmedo-seco a semiárido en el Chaco Seco y en el Espinal.

EI TRB euritermo tropófilo, con una estacionalidad pluviométrica contrastada, está representado en las Yungas y parcialmente en el Chaco Seco. El euritermo xerófilo e hiperxerófilo, con una PVH superior a 5 meses, está presente en la ecorregión de Montes de Sierras y Bolsones.

Respecto a los bioclimas fríos -templados tipo criófilos, se encuentran distribuidos en condiciones de PVH en la Puna (crio xerófilo y crio hiperxerófilo) y en la Estepa Patagónica (crio tropófilo y crio xerófilo). Estas ecorregiones se desarrollan en condiciones bioclimáticas similares pero una en un altiplano, la Puna, y la otra en llanura, con respuestas fisionómicas similares con dominio de herbáceas amacolladas y plantas adaptadas a condiciones de permanencia de manto de nieve. El TRB crio xerófilo también se observa en la ecorregión de Montes de Sierras y Bolsones.

Hay dos situaciones con bioclimas crio mesófilos, con sólo PVT de 1 a 5 meses, en Monte de Llanura y Mesetas y en Pampa y Espinal más meridional en condiciones ombroclimaticas húmedo a subhúmedo en la ecorregión Pampa y semiárido en el Espinal.

En condiciones de medios subpolares con bioclimas micro mesófilas sólo se encuentran las Islas del Atlántico Sur en condiciones de ombroclima subhúmedo seco y en los bosques patagónicos en ombroclima hiperhúmedo a subhúmedo-seco. En este mismo medio pero con PVH, se sitúan los Altos Andes (mesocrio xerófilo e hiperxerófilo en condiciones ombroclimáticas áridas), y en la Estepa Patagónica más meridional con bioclima mesocrio tropófilo semiárido. 
En esta síntesis no están representados actualmente en Argentina los bioclimas del dominio tropical, que aparecen ya en Brasil o Paraguay. En la tabla 3 se muestra la relación entre las ecorregiones de Argentina y los tipos de regímenes bioclimáticos.

Tabla 3. Relación entre las ecorregiones de Argentina y los tipos de regímenes bioclimáticos en los que se distribuyen.

\begin{tabular}{|c|c|}
\hline ECORREGIONES & TRBs \\
\hline 2-Islas del Atlántico sur & Mesocrio mesófilo subhúmedo-seco \\
\hline 3-Altos Andes & $\begin{array}{l}\text { Mesocrio xerófilo árido/ mesocrio hiperxerófilo árido/ hipercrio tropófilo } \\
\text { semiárido/ hipercrio xerófilo árido }\end{array}$ \\
\hline 4-Puna & Crio xerófillo semiárido-árido/ crio hiperxerófilo árido /mesocrio xerófilo árido \\
\hline 5-Seva Paranaense & Euritermo mesófilo húmedo superlativo a medio \\
\hline 6 -Yungas & Euritermo tropófilo subhúmedo-seco \\
\hline 7-Bosques Patagónicos & Mesocrio mesófilo hiperhúmedo-subhúmedo seco \\
\hline 9-Campos y Malezas & Euritermo mesófilo húmedo medio \\
\hline 10-Chaco Húmedo & Euritermo mesófilo húmedo-subhúmedo a subhúmedo-seco \\
\hline 11-Chaco Seco & $\begin{array}{l}\text { Euritermo mesófilo subhúmedo-seco a semiárido/ euritermo tropófilo } \\
\text { semiárido }\end{array}$ \\
\hline 12-Delta e Islas & Euritermo mesófilo húmedo-subhúmedo \\
\hline 13-Espinal & $\begin{array}{l}\text { Euritermo mesófilo húmedo-subhúmedo a subhúmedo seco/ crio mesophyllo } \\
\text { semiárido }\end{array}$ \\
\hline 14-Estepa Patagónica & $\begin{array}{l}\text { Crio tropófilo semiárido a árido/ crio xerófilo árido/ mesocrio tropófilo } \\
\text { semiárido }\end{array}$ \\
\hline 15-Esteros del Iberá & Euritermo mesófilo húmedo inferior a húmedo-subhúmedo \\
\hline 16-Monte de Llanura y Mesetas & Crio mesófilo semiárido/ crio tropófilo semiárido a árido \\
\hline 17-Monte de Sierras y Bolsones & Euritermo xerófilo árido/ euritermo hiperxerófilo árido/ crio xerófilo árido \\
\hline 18- Pampa & $\begin{array}{l}\text { Euritermo mesófilo húmedo inferior a subhúmedo-seco/ crio mesófilo húmedo- } \\
\text { subhúmedo }\end{array}$ \\
\hline
\end{tabular}

Fuente: Elaboración propia.

\subsection{Tipos de Regímenes Bioclimáticos con RCP 8.5 para el escenario 2070}

En el mapa del TRB de escenario futuro de cambio climático se seleccionó el año 2070. En el Quinto Informe del IPCC se han definido 4 nuevos escenarios de emisión denominadas RCP. En cada una de ellas, hay una situación asociada según las emisiones de los gases de efecto invernadero (GEI): un nivel muy bajo (RCP 2.6), dos de estabilización (RCP 4.5 y RCP 6.0) y un escenario con un nivel muy alto de concentración (RCP 8.5). La finalidad de la elaboración de esta cartografía es analizar cómo se verían modificados los actuales TRB con la influencia de estos gases en la atmósfera, en un escenario donde su concentración sea elevada (RCP 8.5). De esta forma, poder planificar estrategias que permitan reducir los gases contaminantes y evitar que este modelo futuro, se convierta en una realidad. 


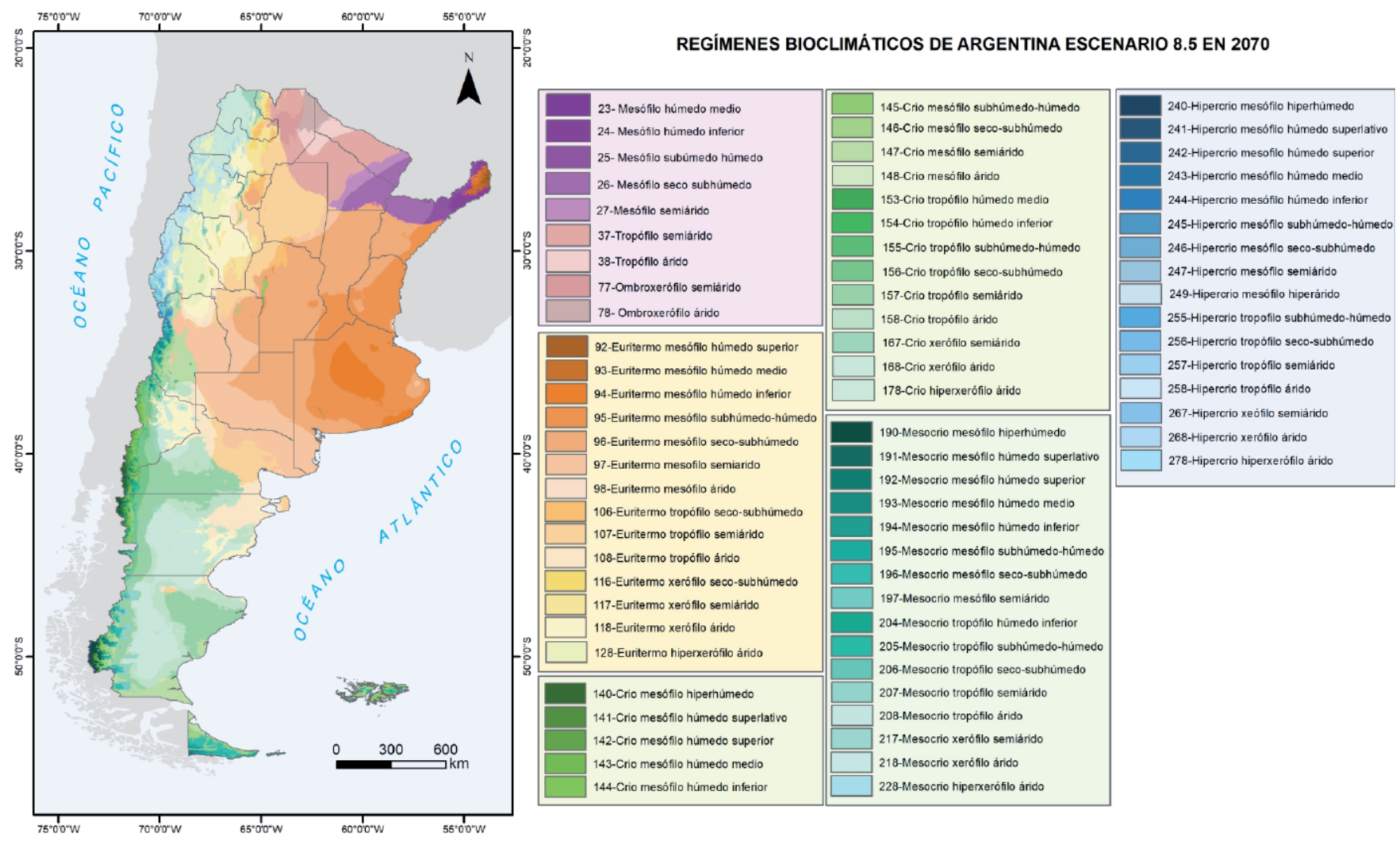

Mapa 3. Tipos de Regímenes Bioclimáticos de Argentina para el escenario de cambio climático con RCP 8.5 - año 2070. Fuente: Elaboración propia.

En el mapa 3 se muestra una totalidad de 19 TRB y 73 TRBs. Los TRB mesófilo, tropófilo y xerófilo (bioclimas tropicales) aparecen en esta cartografía, estando ausentes en las anteriores. Se distribuyen en el Noreste y centro-Norte del país. En el mapa actual (mapa 2), no se visualizan dichos tipos por lo cual se puede inferir que el incremento de los GEI considerando un RCP elevado, daría lugar a un incremento en las temperaturas en dicho sector del país. Según la Secretaría de Ambiente y Desarrollo Sustentable de la Nación (2015) y Camilloni (2018), los cambios en temperatura proyectados para fines del presente siglo indican incrementos en todo el país con valor promedio de $1^{\circ} \mathrm{C}$, aunque en el Noroeste y Patagonia se observarían los máximos cambios con temperaturas que ascienden de $3,5^{\circ} \mathrm{C}$ a $5^{\circ} \mathrm{C}$.

Como producto de la aparición de nuevos TRB al norte del país, los tipos asociados a los TRB euritermófilos se ven desplazados más hacia el Sur, abarcando parte de la Patagonia Norte, en las provincias de Río Negro, Chubut y Mendoza. Sigue predominando como en el Mapa 2 el tipo euritermo mesófilo, debido a su mayor extensión en todo el territorio argentino. Los tipos vinculados a los bioclimas criófilos se extienden principalmente en la Patagonia, cuyo límite norte fue modificado y se limita al sector central y Sur. También en la zona vinculada a la Cordillera de los Andes, en el noroeste del país. En el resto de las provincias de la Patagonia predominan los TRB criófilos y los mesocriófilos e hipercriófilos se encontrarían en el sector de las montañas. Los tipos vinculados a los bioclimas mesocriófilos e hipercriofilos se ven reducidos en su superficie. En el primer caso a sitios específicos de la Cordillera de los Andes, en Tierra del Fuego y en sectores de las Islas Malvinas. Los tipos del bioclima hipercriófilo, solamente se distribuyen en el Oeste del país, relacionados con el sistema montañoso de los Andes, desde Jujuy hasta Mendoza.

Según la Secretaría de Ambiente y Desarrollo Sustentable de la Nación (2015), para el futuro lejano se proyecta un descenso de 10 a $20 \%$ en el oeste de la Patagonia norte y central y un aumento en el centro y Este del país. Se ha observado un incremento en superficie de la xerofilia, que determina la paralización vegetativa de 5 a 8 meses. 


\section{DISCUSIÓN}

Si bien existe un antecedente de regionalización bioclimática en Argentina, este está más enfocado en los aspectos energéticos vinculados con el hábitat (Czajkowski et al., 2009). Entonces, en comparación con lo presentado en este trabajo de investigación se puede mencionar que existen diferencias en cuanto a los métodos utilizados para la obtención de los mapas bioclimáticos, también en las variables climáticas usadas y en la base de datos y finalmente en el nivel de detalle de las categorías detectadas en cada clasificación. Además, el análisis de este artículo, se realizó considerando otros períodos de tiempo, pasado y futuro.

Si se comparan los TRB del Último Máximo Glaciar con la situación actual y el escenario de cambio climático con RCP 8.5 para el 2070, se observan algunas modificaciones significativas. Entre ellos la superficie de los tipos térmicos que fue cambiando en cuanto a su preponderancia en cada período analizado. Mientras que el criófilo fue el de mayor extensión durante el último Máximo Glaciar con 1.526 .738 km², en el mapa actual y en el escenario 2070, es el euritermófilo, el que obtiene mayor predominio, con $1.365 .641 \mathrm{Km}^{2} \mathrm{y}$ 1.598.736 Km² respectivamente. Se observa además una reducción notable del mesocriófilo e hipercriófilo y en menor medida del criófilo entre el primer período y el segundo (Mapa 1 a Mapa 2), tendencia que se mantiene para el escenario del 2070. Por el contrario, aparece el tropófilo como un nuevo tipo térmico que se visualiza con una gran superficie en el año 2070 con una superficie de 147.151,9 km². El euritermófilo, durante este período, incrementa su superficie, aunque no de forma tan significativa como del primero al segundo mapa.

Si se considera el periodo actual con el escenario de cambio climático del año 2070, se observan incrementos y decrecimientos de los TRBs entre ambos períodos. La variación de su superficie se observa en la tabla 4.

El bioclima tropófilo en el dominio tropical para 2070, alcanzaría una superficie de 147.152 km² y el mesófilo $111.146 \mathrm{~km}^{2}$, ubicados ambos al Norte de Argentina, frontera con Brasil y Paraguay. Otros TRBs que se verán ampliados en su área de distribución de forma significativa para el 2070, serían: euritermo mesófilo, tropófilo, xerófilo e hiperxerófilo. Es decir, se incrementan los TRB de categoría tropical y subtropical. Por el contrario, todos los TRBs de las categorías templados-fríos, subpolares y polares sufrirían pérdidas en su superficie. Los que tienen un decrecimiento más elevado en el futuro serían: euritermo ombrófilo, crio ombrófilo, crio mesófilo, crio mesófilo, y crio tropófilo. Los bioclimas crio xerófilo y crio hiperxerófilo aumentan en superficie, siendo esto una anomalía que se explica por la presencia de la Cordillera de los Andes, por aumento de la nivación.

Se destaca en este trabajo las transformaciones espaciales de los regímenes bioclimáticos en los períodos seleccionados, que se pueden explicar como producto del cambio climático. En Argentina, este fenómeno se caracteriza por un incremento en las temperaturas medias anuales, principalmente en la Patagonia, y por la variación en las precipitaciones por regiones, incrementándose en la zona centro-este del país y disminuyendo en la región de Cuyo (Camilloni, 2018). El aumento de los GEI se observa principalmente en la época del Antropoceno, término acuñado por Crutzen \& Stoermer (2000). Estas trasformaciones se han intensificado en los últimos siglos repercutiendo también en los regímenes bioclimáticos. Tonni (2006) afirma que los cambios climáticos durante el Holoceno afectaron la composición y distribución de la biota generando incluso el proceso de extinción de algunos seres vivos. Se puede inferir que desde el Holoceno y hasta el escenario 2070, estos procesos se irán acelerando y generando la necesidad de procesos adaptativos en las plantas y animales, afectando la cantidad de su población y su distribución en el territorio argentino y en algunos casos, determinando su peligro de extinción (Teta et al., 2014). Algunos ejemplos ya analizados con miras al futuro se visualizan en Rodríguez Cravero et al. (2017), en el cual estudiaron la aptitud de hábitat del género Stevia en el noroeste del país comparando su distribución actual con la del escenario 2050 con RCP 2.5 y RCP 8.5. 
Tabla 4. Variación de la superficie absoluta en $\mathrm{Km}^{2}$ de los TRB en los Mapas 1, 2 y 3 de los períodos analizados. El color rosado indica regresión de superficie y el azul progresión.

\begin{tabular}{|c|c|c|c|c|}
\hline \multicolumn{2}{|c|}{ Tipos de Regímenes Bioclimáticos } & Último Máximo Glaciar & Actual & Escenario RCP 8.52070 \\
\hline \multirow{3}{*}{ TROPICALES } & Mesófilo & 0 & 0 & 111146 \\
\hline & Tropófilo & 0 & 44.8 & 147151.9 \\
\hline & Ombroxerófilo & 0 & 0 & 31994.9 \\
\hline \multirow{5}{*}{ SUBTROPICALES } & Euritermo ombrófilo & 305.0 & 1.4 & 0 \\
\hline & Euritermo mesófilo & 400663.2 & 997182.0 & 1024800.0 \\
\hline & Euritermo tropofilo & 220560.7 & 295782.2 & 332234.0 \\
\hline & Euritermo xerófilo & 12062.7 & 60040.4 & 182215.6 \\
\hline & Euritermo hiperxerófilo & 335.5 & 12635.7 & 59486.7 \\
\hline \multirow{5}{*}{ TEMPLADOS-FRÍOS } & Crio ombrófilo & 0 & 5.6 & 0 \\
\hline & Crio mesófilo & 737764.5 & 270614.4 & 139795.6 \\
\hline & Crio tropófilo & 317566.0 & 531931.4 & 350157.5 \\
\hline & Crio xerófilo & 421372.7 & 259765.1 & 266020.3 \\
\hline & Crio hiperxerófilo & 50035.2 & 19859.7 & 33978.7 \\
\hline \multirow{4}{*}{ SUBPOLARES } & Mesocrio mesófilo & 23149.5 & 101529.4 & 54468.4 \\
\hline & Mesocrio tropófilo & 183396.5 & 119784.0 & 28469.7 \\
\hline & Mesocrio xerófilo & 664457.7 & 67412.1 & 23440.2 \\
\hline & Mesocrio hiperxerófilo & 1769.0 & 11368.0 & 4654.3 \\
\hline \multirow{4}{*}{ POLARES } & Hipercrio mesófilo & 401746.0 & 26100.9 & 14044.8 \\
\hline & Hipercrio tropófilo & 103334.0 & 40289.9 & 12459.3 \\
\hline & Hipercrio xerófilo & 82273.7 & 18431.0 & 6103.3 \\
\hline & Hipercrio hiperxerófilo & 1189.5 & 1091.3 & 3286.5 \\
\hline
\end{tabular}

Fuente: Elaboración propia.

Como se mencionó anteriormente no existen antecedentes en el país sobre este tipo de estudios y por lo tanto no se puede realizar ninguna comparación. Sin embargo, en relación con el caso de Paraiba se pueden visualizar algunas similitudes, más allá de las diferencias en la distribución de los regímenes bioclimáticos y en la escala de análisis de esta región de Brasil con la Argentina. Según Cámara et al. (2022), en Paraiba se observaron cambios en los TRB durante los tres periodos analizados. Si se hace hincapié en el mapa actual en relación con el del año 2070, en el primero se registra la presencia de 9 TRB, mientras que en el segundo el número se reduce a 4 . En el escenario futuro se observan subtipos más áridos, desapareciendo los húmedos y subhúmedos. Esta situación modificaría completamente el área de distribución de las plantas. En el caso de Argentina, los cambios más significativos se muestran sobre la base de la condición térmica, incrementándose los tipos los medios tropicales y subtropicales y disminuyendo la superficie de los medios templados-fríos y subpolares. Este nuevo patrón de los regímenes bioclimáticos en el escenario futuro de cambio climático también afectaría el área de distribución de las plantas y consiguientemente de las formaciones vegetales de Argentina. 


\section{CONCLUSIONES}

La generación de mapas bioclimáticos es esencial para definir la distribución de los seres vivos en un territorio. En especial, la metodología de TRB (Cámara Artigas et al., 2020) es un gran aporte en la investigación sobre esta temática ya que tiene como ventajas su utilización y aplicación en distintas escalas de análisis (nacional, regional, provincial, etc.) y en diferentes temporalidades, abarcando desde Último Máximo Glaciar hasta escenarios futuros de cambio climático. Su representación cartográfica permite ver rápidamente la información sobre los regímenes bioclimáticos de un territorio y establece modelos para poder definir posibles escenarios para el futuro bajo elevadas concentraciones de GEI.

El estudio realizado muestra los cambios y dinámica de los tipos bioclimáticos desde el Último Máximo Glaciar hasta la actualidad, y desde la situación de esta hasta un escenario de cambio climático con RCP 8.5 en 2070. El resultado es una fase de calentamiento holocena hasta la actualidad en la que retroceden los bioclimas de medios subpolares (mesocriófilos) y templados fríos (criófilos) y aumentan la superficie de los subtropicales (euritermófilos) y en 2070 aparecen los tropicales (mesófilos y tropófilos) debido al calentamiento general por la subida de hasta $3^{\circ} \mathrm{C}$ para dentro de 50 años (hasta 2017) según RCP 8.5, que es el escenario utilizado.

Esta metodología permite constatar los cambios cuaternarios en los bioclimas, y por lo tanto en las formaciones vegetales y ecorregiones, a partir del conocimiento actual de la distribución de estas conforme a los tipos de regímenes bioclimáticos. De la misma manera se pueden inferir los cambios futuros a partir de la información disponible de temperaturas y precipitaciones mensuales en la base de datos continua climática de Wordclim y las relaciones de los TRB actuales con la vegetación actual tal como se refleja en la Tabla 3. La validez de este método ha sido contratada con la realización del mapa mundial de la situación actual (Cámara, 2004; Cámara et al., 2020, Cámara et al. 2022), o su aplicación a lugares concretos a nivel nacional o regional.

En Argentina, no existe una clasificación actualizada de los regímenes bioclimáticos así como tampoco una cartografía que sea temporal, con lo cual este trabajo constituye un avance fundamental en esta temática. Puede ser utilizado para la explicación de las áreas de distribución de las especies pasadas, presentes y futuras. Se pueden derivar estudios sobre diferentes problemáticas a partir de la aplicación de esta metodología en regiones y provincias del país. Por ejemplo, se pueden combinar los regímenes bioclimáticos con las ecorregiones del territorio para conocer su correspondencia y de esta forma explicar las variaciones en la fisonomía de la vegetación entre distintas ecorregiones e inclusive en el interior de cada una de ellas. Por otra parte, conocer mediante el modelado las condiciones bioclimáticas futuras de cambio climático permite activar líneas de trabajo de planificación en pos de mitigar los efectos adversos generados por los elevados niveles de concentración de GEI en la atmósfera.

\section{Agradecimientos y financiación}

Este trabajo se realizó en el marco del proyecto de investigación Geografía física aplicada al estudio de la interacción sociedad-naturaleza. Problemáticas a diversas escalas témporo-espaciales (24/G078). Este es dirigido por la Dra. Alicia M. Campo y se encuentra subsidiado por la Secretaría de Ciencia y Tecnología de la Universidad Nacional del Sur. También al apoyo del Consejo Nacional de Investigaciones Científicas y Técnicas (CONICET).

\section{Declaración responsable y conflicto de intereses}

Los autores declaran que no existe ningún conflicto de interés con relación a la publicación de este artículo. Los dos autores han participado en la revisión bibliográfica, la aplicación de la metodología, la elaboración de la cartografía y la redacción del artículo. 


\section{REFERENCIAS}

Bagnouls, F. \& Gaussen, H. (1963). Los Climas Biológicos e Sua Classificacao. Boletim Geográfico, 76, 545-566. Bailey, R.G. (2009). Ecosystem Geography. From Ecoregions to Sites. Springer. https://doi.org/10.1007/978-0-387-89516-1

Bruniard, E.D. (2000). Los Regímenes Climáticos y la Vegetación Natural. Aportes para un modelo fitoclimático mundial, Academia Nacional de Geografía. Academia Nacional de Geografía.

Cain, S.A. (1944). Foundations of plant geography. Harper and Brothers.

Cámara Artigas, R., Díaz del Olmo, F. \& Martínez-Batlle, J. R. (2020). TBRs, a methodology for the multi-scalar cartographic analysis of the distribution of plant formations. Boletín de la Asociación de Geógrafos Españoles, 85(2915), 1-38. https://doi.org/10.21138/bage.2915

Cámara R. (2004). Escalonamiento Bioclimático, Regímenes Ecodinámicos y Formaciones Vegetales de la Isla de la Española en República Dominicana. En: Panareda (Ed.) Estudios en Biogeografía 2004: Libro Homenaje a José Manuel Rubio Recio y Jesús García Fernández (p. 39-55). Terrassa, Aster.

Cámara Artigas, R., De Souza, B.I. \& Porto De Lima, R. (2022). Climatic changes and distribution of plant formations in the state of Paraiba, Brazil. Cuadernos de Investigación Geográfica, 48. En prensa. https://doi.org/10.18172/cig.5044

Camilloni, I. (2018). Argentina y el cambio climático. Ciencia e Investigación, 5, 5-10. http://aargentinapciencias.org/ wp-content/uploads/2018/11/1-Camilloni-cei68-5-2.pdf

Compagnucci, R.H. (2011). Atmospheric circulation over Patagonia since the Jurassic to present: a review through proxy data and climatic modeling scenarios. Biological Journal of the Linnean Society, 103(2), 229- 249. https://doi.org/10.1111/j.1095-8312.2011.01655.x

Crutzen, P.J. \& Stoermer, E.F. (2000). The 'Anthropocene". Global Change Newsletter, 41, 17-18. https://doi.org/10.17159/ sajs.2019/6428

Czajkowski, J.D., Gómez, A., Martini, I. \& Rosenfeld, Y. (2009). Regionalización bioclimática de Argentina mediante el uso de técnicas multivariadas y SIG. [Comunicación en congreso], VI Encuentro de Geógrafos de América Latina (EGAL). Buenos Aires, Argentina.

Czajkowski, J.D. \& Rosenfeld, E. (1992). Regionalización bioclimática de la provincia de Buenos Aires. [Comunicación en congreso]. XV Reunión de Trabajo de la Asociación Argentina de Energía Solar (ASADES). Catamarca, Argentina.

Doyle, M. (2019). El clima en Argentina, su variabilidad, cambio y posibles escenarios futuros. Revista Argentina de producción animal, 39, 31-41. https://ppct.caicyt.gov.ar/index.php/rapa/article/view/16608/pdf

Entrocassi, G.S., Hormigo, D.F., Gavilán, R.G. \& Sánchez-Mata, D. (2014). Bioclimatic typology of Jujuy Province (Argentina). Lazaroa, 35, 07-18. https://doi.org/10.5209/rev_LAZA.2014.v35.42366

Fernández González, F. (2004). Bioclimatología. En J. Izco (Coord.) Botánica (p. 715-794). Mc Graw-Hill Interamericana.

Fick, S.E. \& Hijmans, R.J. (2017). WorldClim 2: new $1 \mathrm{~km}$ spatial resolution climate surfaces for global land areas. International Journal of Climatology, 12, 4302-4315. https://doi.org/10.1002/joc.5086

Fidalgo Hijano, C. \& Muñoz Jiménez, J. (2003). Nuevas aportaciones para un modelo fitoclimático mundial. Ería. Revista Cuatrimestral De Geografía, 60, 133-137. https://doi.org/10.17811/er.0.2003.133-137

García, E. (1964). Modificaciones al Sistema de Clasificación Climática de Köppen (para adaptarlo a las condiciones de la República Mexicana (5 $5^{a}$ ed.). Instituto de Geografía, UNAM.

González Loyarte, M.M., Menenti, M. \& Diblasi, A. (2009). Mapa bioclimático para las Travesías de Mendoza (Argentina) basado en la fenología foliar. Revista de la Facultad de Ciencias Agrarias, 41(1), 105-122. http://revista.fca.uncu.edu. ar/images/stories/pdfs/2009-01/T41_1_10.pdf

Hausfather, Z. \& Peters, G. (2020). Emissions the 'business as usual' story is misleading. Nature, 577(7792), 618-20. https://doi.org/10.1038/d41586-020-00177-3

Hernández Cerda, M.E., Ordoñez Díaz, M. \& Giménez de Azcárate, J. (2018). Análisis comparativo de dos sistemas de clasificación bioclimática aplicados en México. Investigaciones Geográficas, 95, 1-14. https://doi.org/10.14350/rig.57451

Hijmans, R.J., S.E. Cameron, J.L. Parra, P.G. Jones \& A. Jarvis, (2005). Very high resolution interpolated climate surfaces for global land areas. International Journal of Climatology, 25, 1965-1978 https://doi.org/10.1002/joc.1276

Holdridge, L. R. (1947). Determination of world plant formation from simple climatic data. Science, 105, 267-268. http:// dx.doi.org/10.1126/science.105.2727.367 
IPCC. (2013). Climate Change 2013: The Physical Science Basis. Contribution of Working Group I to the Fifth Assessment Report of the Intergovernmental Panel on Climate Change. Stocker, T.F., D. Qin, G.-K. Plattner, M. Tignor, S.K. Allen, J. Boschung, A. Nauels, Y. Xia, V. Bex and P.M. Midgley (eds.). Cambridge University Press, Cambridge, United Kingdom and New York, USA.

Ministerio de Ambiente del Ecuador. (2013). Modelo bioclimático del Ecuador continental. Para la representación cartográfica de ecosistemas del Ecuador continental. Ministerio de Ambiente del Ecuador.

Montero de Burgos, J.L. \& González, J.L. (1974). Diagramas bioclimáticos. ICONA.

Morello, J., Metteucci S.D., Rodríguez, A.F. \& Silva, M.E. (2012). Ecorregiones y complejos ecosistémicos argentinos. FADU y GEPAMA. Buenos Aires. Orientación Gráfica Editora.

Ninahuaman Mucha, N. (2016). Investigación bioclimática del Perú, un aporte a la educación para mitigar los efectos del cambio climático. [Tesis de Magister, Universidad Autónoma de ICA]. Repositorio Institucional Universidad Autónoma de ICA. http://repositorio.autonomadeica.edu.pe/handle/autonomadeica/66

Peroné, D. \& Cannelli, N. (1987). Clasificación bioclimática de la región NEA. [Comunicación en congreso]. Actas de la 12a. reunión de trabajo de ASADES. Mendoza, Argentina.

Ponce, F. \& Rabassa, J. (2012). La plataforma submarina y la costa atlántica argentina durante los últimos 22.000 años. Ciencia Hoy, 127, 11-17. https://www.cienciahoy.org.ar/ch/In/hoy127/Plataformasubmarina.pdf

Rivas Martínez, S., Rivas Sáenz, S. \& Penas, A. (2011). Worldwide bioclimatic classification system. Global Geobotany, 1, 1-634.

Rodríguez Cravero, J., Grosi, M., Fuentes-Castillo, T. \& Gutiérrez, D.G. (2017). Cambio climático y modelado de distribución de especies de Stevia (Asteraceae) en el noroeste de la Argentina. Ecología Austral, 27, 462-473. https://doi. org/10.25260/EA.17.27.3.0.588

Rolla, A.L., Nuñeza, M., Guevarad, E., Meirad, S., Rodríguez, G. \& Ortiz de Zárate, M.I. (2018). Climate impacts on crop yields in Central Argentina. Adaptation strategies. Agricultural Systems, 160, 44-59. https://doi.org/10.1016/j. agsy.2017.08.007

Secretaría de Ambiente y Desarrollo Sustentable de la Nación. (2015). Tercera Comunicación Nacional el Gobierno de la República Argentina a las Partes de la Convención Marco de las Naciones Unidas sobre Cambio Climático. Secretaría de Ambiente y Desarrollo Sustentable de la Nación. https://unfccc.int/resource/docs/natc/argnc3s.pdf

Steffena, W., Rockströma J., Richardson K., Lentond T.M., Folkea, C. Diana Livermanf D., Summerhayesg C.P., Barnoskyh, A.D., Cornella S.E., Crucifixi, M. Dongesa, J.F., Fetzera, I., Ladea, S.J., Scheffer M., Winkelmannk, R. Schellnhubera, H.J. (2018). Trajectories of the Earth System in the Anthropocene. PNAS, 115(33), 8252-8259. https://doi.org/10.1073/ pnas. 1810141115

Teta P., Formoso A., Tammone M., Tommaso D. C. de, Fernández F. J., Torres J. \& Pardiñas U.F.J. (2014). Micromamíferos, cambio climático e impacto antrópico: ¿Cuánto han cambiado las comunidades del sur de América del Sur en los últimos 500 años?. Therya, 5 1, https://doi.org/10.12933/therya-14-183

Thornthwaite, C.W. \& Mather, J.R. (1955). The Water Balance. Climatology, 8, 1-104. https://doi.org/10.1017/ CBO9781107339200.011

Tonni, E. (2006). Cambio climático en el holoceno tardío de la Argentina. Una síntesis con énfasis en los últimos 1000 años. Folia Histórica del Nordeste, 16, 187-195. https://doi.org/10.30972/fhn.0163429

Tuhkanen, S. (1980). Climatic parameters and indices in plant geography. Acta Phytogeographica Suecica, 67, 1-108. http://dx.doi.org/10.30972/fhn.0163429

Uribe, J.M., Cabrera, R., De la Fuente, A. \& Paneque, M. (2012). Atlas bioclimático de Chile. Santiago de Chile: Facultad de Ciencias Agronómicas. http://repositorio.uchile.cl/handle/2250/178567

Walter, H. (1997). Zonas de Vegetación y clima. Omega.

Ward, J.D., Mohr, S.H., Myers, B.R., Nel, W.P. (2012). High estimates of supply constrained emissions scenarios for longterm climate risk assessment. Energy Policy, 51, 598-604. https://doi.org/10.1016/j.enpol.2012.09.003

Zazulie, N., Rusticucci, M. \& Raga, G. (2017). Regional climate of the Subtropical Central Andes using high-resolution CMIP5 model. Climate Dynamics, 51, 2913-2925. https://doi.org/10.1007/s00382-017-3560-X

Zhang Y., Feng F., Zhang M., Ren H., Bai J., Guo Y., Jiang H., Kang L. (2016). Residual coal exploitation and its impact on sustainable development of the coal industry in China, Energy Policy, 96, 534-541. https://doi.org/10.1016/j. enpol.2016.06.033 